\section{EXOPHTHALMOS IN BRAIN TUMOR}

\section{WITH REPOR'T OF EIGIIT CASES *}

\section{T. H, WEISLNBURG, M.D.}

l'rofessor of Clnical Neurology, Medico-Chirurgleal College; Nicurologlst to the Plithadelphin Generul Hospital

\section{P'IILADLLL'HIA}

little emplasis has been placed on the occurrence of exophthalmos in brain tumor if the literature on this subject is taken into consicleration, and yot in the clinical description of such cases it is not infequently mentioned. Iy attention was particularly directed to this subject by a number of cases of brain tumor in which exuplithalmos was a prominent symptom, and in a cursory examination of the listories of patients with brain tumor which I have olsserved during the last five years, protrusion of the eyeballs was present either unilaterally or hilaterally in ten, five with necropsy. Of these, eight are here reported.

With the exception of isolated instances, the only extensive papers on the subject are by G. Flateau, ${ }^{1}$ and, to a less extent, by Rosenblath, ${ }^{2}$ who together collected twenty-four ases of tumor accompanied by exophthalmos. The neoplasms were located in practically every pat of the cranial cavity: Six were in the frontal lobe, turo in the hrpophysis, one in the pineal gland, one a basal tumor involving the pons, medulla and cerebellum, two in the cerebello-pontile angle, one in the pons, two in the cerebellum, two in the temporal lobe, one in the right lateral ventricle, and six were instances of internal hydrocephalus.

'The nature of the growths varied, consisting of glioma, sarcoma, neuroma, endothelioma, psammoma, and echinococcus, and it is evident from this that the character of the tumnor probably had no direct bearing on the occurrence of exophthalmos any more than that certain forms of tumor may give metastasis.

In some of these cases the orbits were examined, and in other's the nature of the growths was such as to preclude the opinion that there was metastasis to the orbital cavity. Exophthalmic goiter was excluded in all.

'The exophthalmos was bilateral in all the cases of internal hyclrocephalus and in those tumors in which the lesion involved both sides of the brain, with the exception of one basal tumor. In the unilateral growths the ocular protrusion was one-sided. In all the protrusion was on the side of the lesion, with the exception of one cerebellopontile tumor.

With the exception of the above no attempt is made to quote the cases from the literature. Of my personal (ases only a brief abstract of the histories is given.

\section{REPORT OF CASES}

Case 1.-Gliomatous tumor of lateral and third ventricle compressing and implicating one optic thalamus with bilateral exophthalmos.-Previously reported. ${ }^{3}$ The patient was Miss M-n, a girl about 20, who presented, as one of t':e early symptoms, a tendency to write in a perpendicular direction instead of horizontally, this being followed by gradual loss of vision. Examination showed a marked bilateral choked disk. The symptoms of intracranial pressure were very prominent. When she was eximined by me, she lad bulging of both eyeballs, with ptoxis and deviation of the eyes in a downward direction, paralysis of associated ocular : movement, and of convergence upward, less to the right and left, and least dow'u-

* Rend beforir the Section on Ophthalmology, College of l'hysiciuns, April, 1810

1. Flateau, G.: Deltsoh. Arch. f. klln. Med., 1908, lexvil, 433.

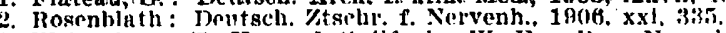

3. Telsenburg. 'T. H., nnd Gulifoyle, W. F.: Kev. Neurol. and Esychlat. June, 1010, p. 325 . wards. At necropsy there was found a tumor of the left lateral ventricle, which grew into the third through the foramen of Monro, and compressed the optic thalanus. All the symptoms which. she presented were readily explained with the exception of the exophthalmos.

The tumor was a vaseular glioma. In the process of its growth from the lateral to the third ventricle it compressed the choroid plexus, the velum interpositum and the veins of Galen, and it is probuble that it caused a stasis in the cavernous sinus, although unfortunately no orbital examination was made. She had, however, a congestion of the veins in the eyelid, with edema and great pulsation of the vessels of the neck (Fiin. 1 ).

CAsE 2.-T'umor of the third ventricle, compressing both optic thalami with mild exophthalmos.-Whe patient, Miss $C_{-}-\mathrm{s}$, was a girl of 17, who had marked pressure symptoms of brain tumor with choked disk of 5 diopters. She had besides wenk. ness of both external recti, paralysis of associatel ocular movement upwards, with loss of convergence in the same direction, and a drunken, ataxic gait, with incoordination of the limbs. At necropsy an extensive gliomatous tumor was found in the third ventricle, compressing both optic thalami and extending downward and bisecting the cerebral peduncles. In this case the orbit was not examined, but there was no reason to suspect metustasis.

Case 3.-Clinically diagnosed as tumor of the third ventricle with wilateral cxophthalmos,-The history of this patient (Miss $M--\%$ ) is very similar to that of Patient 2, but unfortunately no necropsy was permitted. It was that of a girl of 27, who had choked disk of 6 diopters in the right eye and 4 in the left, with marked headache, nausea, vomiting, vertigo, temporary attacks of loss of visiom, and a moderate prominence of the right eye. Examination demonstrated weakness of associated ocular movenent and of convergence upwards, and irregular nystagmus when attempting to look in the same direction; distinct cerebellar gait and incoordina. tion, more marked on the left. The symptoms were indicative of a possible tumor in the third ventricle, as they resembled very much the case cited above. It is noticenble that the exophthatmos was on the side of the greater swelling of the optic nerve.

CASE 4.-Clinically diagnosed as tumor in the third ventriele. Bilateral exophthalmos.-The patient, $\mathrm{R}-\mathrm{L}$, a boy of 12 , had cerebrospinul purulent meningitis (?) from which he recovered. Two months afterward lie began to have headacie, dizriness, nausea, vomiting, dimness of vision, weakness, and a stakgering gait. Examination showed paralysis of associated ocular movement upward and some impairment to the left, with disturbance of convergence in the same directions, wenkness in the right face, arm and leg with l3abinski reflex, and protrusion of both eycballs, which was equally marked on botl sides, and murked choked disk. Unfortunately no necropsy was obtained.

Cass: 5.-Clinically diagnosed as glioma of the pons ant. peduncle. Bilateral exophthalmos.-The putient $\mathrm{C}-\mathrm{y}$ was a boy of 14 , who began with headache, nausea, vomiting, diplopir and difliculty in walking, which brought him to the Will's Eye Hospital, where it was found that he had a bilaternl marked clioked disk of 0 diopters. Examination demonstrated bulging of both eyeballs, more of the right, with paralysis of associated ocular movement and of convergence upward and to the left, which later also involved associated movements to the right. Besides, there was a distinct cerebellar gait, with weukness of the right arm and leg and peripheral weakness of the left seventh nerve. The symptoms were indicative of a gliomatouh tumor in the upper part of the pons and peduncle, jrincipally on the left side. It was noticeable that the exophthalmos was greater on the right, althongh the symptoms were indicative of a greater tumor on the left.

('ASE 6.-Glioma involving the posterior part of the medulla, pons and cerebral perluncle. Unilateral exophthalmos.--This case has already been reported." The patient, $\mathrm{R}-\mathrm{y}$, was 44

4. Weisenburg, T. II.: IExtenslve Gllomatous I'umor Involving the crebellum and the lostertor Portons of the Medulla, Poos and (e" bal leduncle aud the Hostertor limb of One Internal Capsubs Thi: Jolkxal A. M. A., Dec. 18, 1009, p. 2086. 
years of age, and gradually developed general pressure and local symptoms of a right cerchellar tumor, with a choked disk of abont 3 diopters. He was operated on over the right cerebellar area, but nothing was found. While before the operation there was only a partial weakness of the right third and of the sixth, some diminution of hearing in both ears and a little difficulty in articulation, after it there gradually developed a protrusion of the right eyeball and incrensed veakness in the distribution of the right third, and later paralysis of the right motor and sensory fifth, seventh and twelfth. l3esides there was difliculty in articulation and swallowing. At necropsy, an extensive gliomatous tumor was found which involved practically all portions of the cerebellum, but especially the vermis in its inferior part and the left lateral lobe. It also extended diflusely into all the cerebellar peduncles, the posterior portions of the medulla almost as low down as the decussation, the posterior part of the pons and cereloral peduncles, and extended into the posterior portion of the left optic thalamus and internal capsule.

In this case the exophthalmos was unilateral, and on the side on which the cerebellar exploration was performed, and developed after the operation. It is to be noted that the symptoms of crunial nerve and cerebellar involvement gradually developed and becume much more marked after the operation and coincidentally with the exophthalmos, and were explained loy the growth of the tumor. There was no metastasis to the orbit.

( ASE 7.-Basal tumor in posterior cranial fossa. Bilateral cxophthalmos.-'The patient, Miss $\mathrm{M}-\mathrm{C}$, was a young womu of 20, whom I first saw in the Will's Eye Hospital and subsecquently transferred to my wards in the Philadelphin General llospital. She had all the pressure-symptoms of brain tumor, with marked choked disk, cerebellur gait, incoordination, central weakness of the right seventh, motor fifth and tongue, wenkness of all the limbs, possibly more of the right, with incrensed reflexes. Very early she developed exophthılmos, more marked on the left, and there was besides immobility of the left eyeball with ptosis, the only movement possible being a slight movement downward and inwurd. The right eye could be moved downward, but not well in an upward direction. The patient died in the service of Dr. Spiller, who found a very large tumor in the posterior cranial fossa. 'The occipital lobes had been pushed apart and the cerebelum displaced almost to a right angle with the brain stem.

(AsE 8.-Sarcoma, compressing the temporal lobe, optio chiasm and posterior orbital bones. Unilateral exophthalmos. -This patient, Mrs. S-a, whose case was reported a year ago by Dr. de Schweinit\% before the Section on Ophthalmology. was a woman of 63 , who, in 1900 , injured the right orbit, but not very severely. Eight months afterward she began to have a bulging of the right eye, which from then on progressed. Dr. Wendell Reber studied her for a long time, and he states that there were never present ocular palsies. She had optic atroply. The necessory sinuses were always normal. $X$-ray examination was negative. Eight yenrs after the beginning of the exophthalmos, she was admitted to my wards in the Philadelphia Hospital, having then general epileptic convulsions, which were followed by temporary left hemiplegia. She never had any general symptoms of brain tumor. She died eight years after the beginning of the exophthalmos (Fig. 2).

At necropsy a tumor, a sareoma, was found resting in the middle cranial fossa, it being about the size of a small orange. It compressed the orbital bones, the orbital surface of the temporal, the posterior part of the orbital surface of the frontal and the extreme anterior part of the cerebellar lobe. It also deviated and pressed on the optic chiasn, displacing it slightly to the opposite side, and also pressed on the foot of the cerebral peduncle (Fig. 3 ).

Dr. de Schweinitz, who was present at the autopsy, does not believe that the exophthalmos was the result of pressure on the cavernous sinus, although he cannot prove that it was not the result of this. He carefully dissected the specimen and

5. Splller, Whllam G.: A Further Contribution to Jallintive Operations for Brain 'Tumor, Tre Joukxal A. M. A., Jan. 23, 1008 , p. 277 there was no direct pressure on the simus, nor was there on any of the nerves passing into llo orbit. He says, however, that it is perfectly possible that the exophthalmos might have lad such an origin. There was, he further states, a rather hard edena of the posterior tissues of the orbit.

In view of the autopsy, I do not know that it is possible to explain the exophthalmos in any other way than from a purely local cause, and the only local cause that can be given is pressure on the cavernous sinus, which possibility Dr. de Schweinitz himself admits. In support of this, the second, third, fourth and sixth nerves on this side were found diseased, this being especially true of the second, showing that pressure was exerted on it. Hesides, the optic chiasm was compressed and deviated to the other side and the foot of the cerebral peduncle was distorted.

\section{IREATRKS}

In a summary of my cases it is noticealle that in four, Cases $1,2,3$ and the the diagnosis was tumor in the third ventricle, and in two, Cases 5 and 6, a glioma which involved both the pons and peduncle. In Case 7 the growth was in the posterior cranial fossa and was of such size that it compressed the occipital lobes, and twisted the cerebetlum on its axis. In Case 8 tinere was a sarcoma in the anterior portion of the middle fossa which directly pressed on the orbit and the exophthalmos was undoubtedly the result of pressure on the cavernous sinus.

In all, the intracranial pressure was extreme, as indicated by the prominence of the headache, nausea, vomiting, rertigo and especially the choked disk. It is evident also that in the first six there was interference with the flow of the cerebrospinal fluid, inasmuch as there was either direct involvement of the third ventricle or pressure on the aqueduct of Sylvius.

'The exophthalmos was bilateral in five and unilateral in three. In those in which it was milateral either the lesion or the intracranial pressure was greater on the same side. In one, Case 3, there was supposedly a tumor in the third ventricle and the exophthalmos was present on the side of the greater swelling of the optic nerve. It is possible that protrusion of the left eyeball might have developed later. In the second, Case 6, the exophthalmos developed, after the exploratory operation, on the same side, and coincidentally with the development of other cranial symptoms. In the last, Case 8, the exophthalmos was the result of pressure of the tumor directly on the cavernous sinus.

In those in which the exophthalmos was bilateral the lesion was also bilateral and with the exception of one, Case 5, there was a greater prominence of the eveball on the side of the greater involvement. 'The grade of the exophthalmos varied, it being marked in some and mild in others. In all it was progressive and came on early.

These observations agree with the collected cases quioted.

'l'he conclusion, then, which can be drawn from the above is that exoplithalmos occurs in brain tumor and especially in those cases in which there is great intracranial pressure and interference with the flow of the cerebrospinal fluid.

'The question naturally arises how these factors produce exophthalmos. It is well known, especially through the physiologic studies of Leonard Hill, that the earliest manifestation of increase in intracranial pressure is engorgement of the venous circulation, this producing the familiar dilatation and tortuosity of the retinal vessels. There are, however, as has been mentioned by Cushing, other external evidences equally characteristic,

6. Osler's Modern Mediclue, vill, 431 
although less often commented on, which consist in dilatation of the vessels of the scalp and particularly the smaller venules of the eyelids.

If in addition to the above there is exerted pressure on the cavernous sinus, thrombosis of which is the common cause of exophthalmos, the explanation is not at all difficult. Such was the fact in my cases, for in all there was direct pressure on the cavernous sinus. In the first f'our the tumor was in the third ventricle and pressure must have been exerted on the optic chiasm and basal

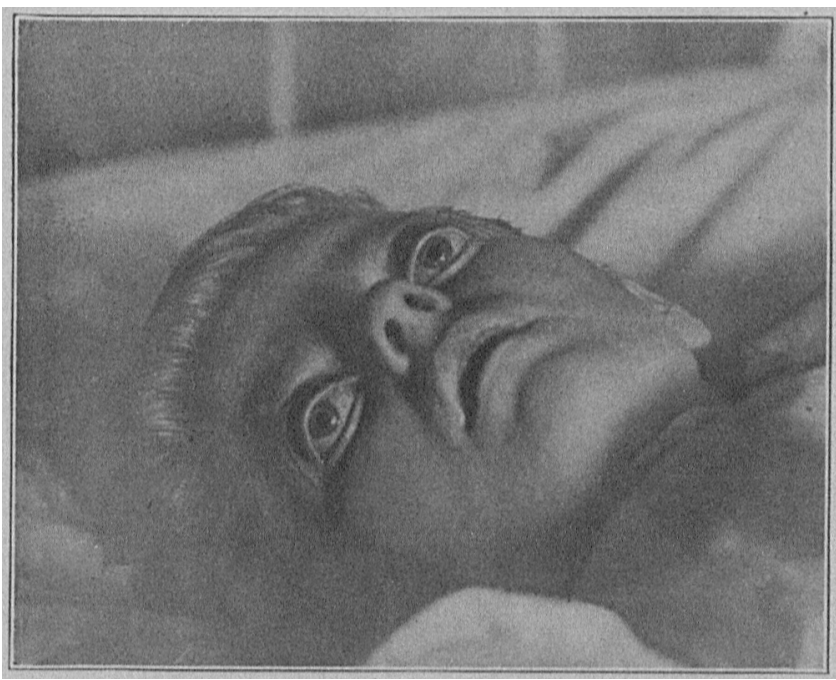

Fig. 1.-Exophthalmos in tumor of the third ventricle. Case 1.

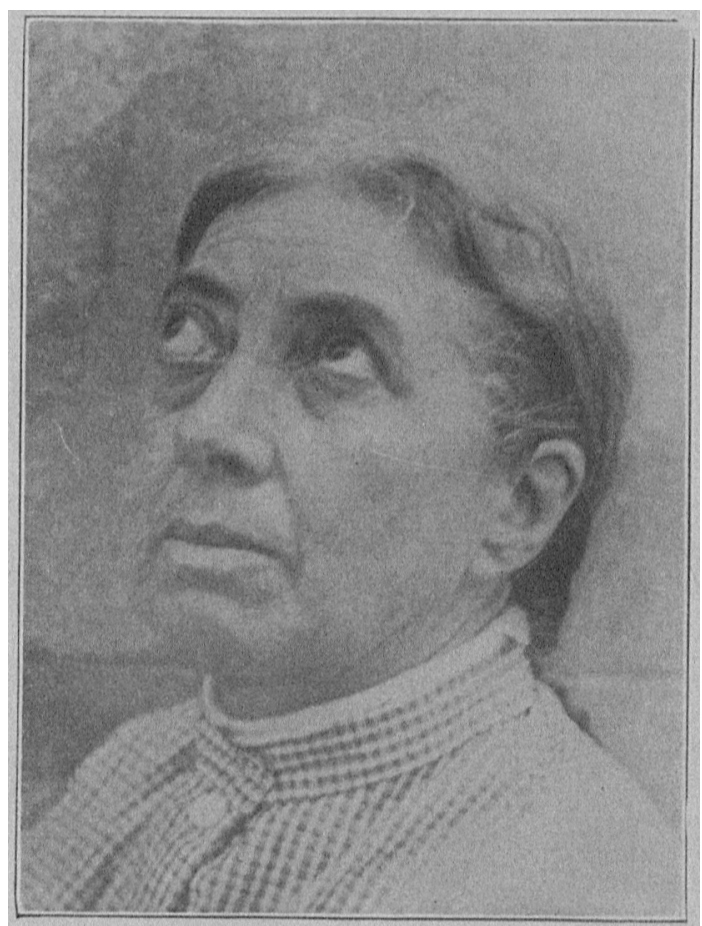

Fig. 2.-Unilateral exophthalmos from tumor in the wlddle fossa case 8.

sinuses, while in Cases 5 and 6 there was obstruction of the cerebrospinal fluid in the aqueduct of Sylvius, this undoubtedly causing dilatation of the third ventricle. In the last two there was direct pressure on the sinus by the timor.

Slatcau advanced a very plausible theory for the occurrence of exophthalmos in those anses in which there is no direct pressure on the eavernous sinus. According to him the blool of the orbit under normal conditions is not depleted by the facial vein but by the cavernous sinus, and the outflow can be interrupted cither directly by pressure on the ophthalmic vein of indirectly from a distant sinus; and tumors, for example, of the posterior cranial fossa can so obstruct the outflow of the blood in their own sinus that a portion must flow out by means of the cavernous sinus. Therefore, the back flow of the blood through the eye will be made so difficult that there will result a stasis in the orbit, this producing exophthalmos.

It is noteworthy, however, that in the material at my disposal, which consisted of the records of about 75 cases of brain tumor, exophthaimos occurred only in the cases mentioned and was readily explained by direct pressure on the cavernous sinus.

\section{CONCLUSIONS}

The conclusions which can be drawn from the study of this subject are:

1. Exophthalmos accompanies brain tumor more f'requently than is generally supposed.

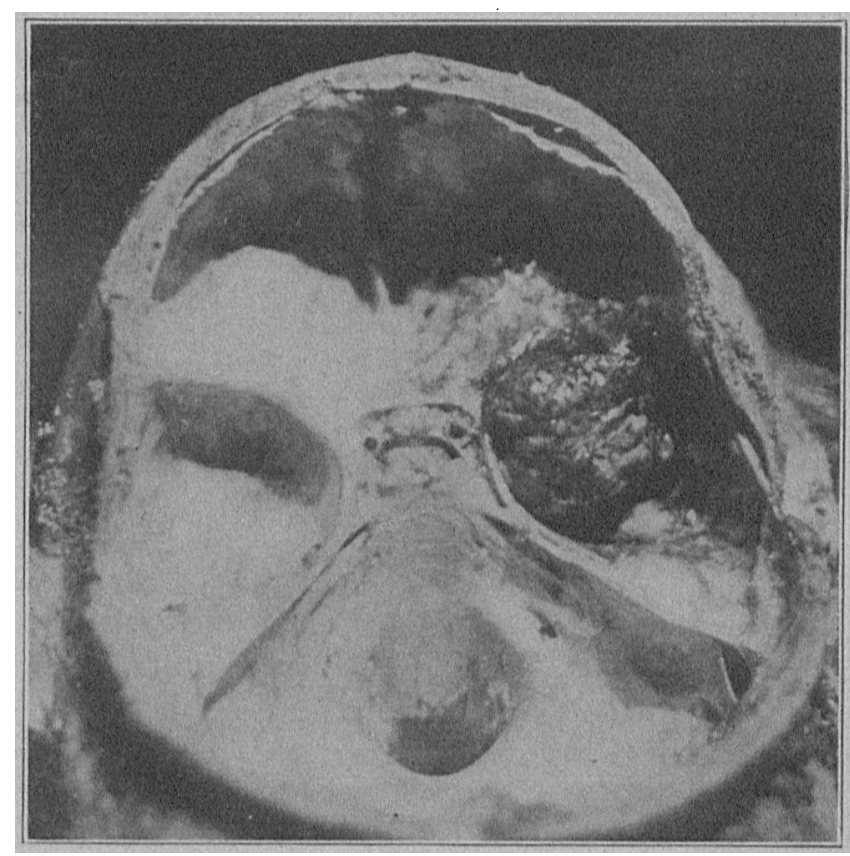

Fig. 3.--'lumor in middlo fossa, producing unilateral exophthnimos in pulient whose photograph is shown in Fig. 2 .

?. It occurs only in those cases in which there is great intracranial pressure, especially when there is in addition direct interference with the normal flow of the cerebrospinal fluid.

3. Exoplitlalmos is produced by direct pressure on tho cavernous sinus.

t. Its presence is of some elinical value inasmuch as unilateral exophthalmos is nearly always indicative of an intracranial lesion on the same side.

5. In those cases in which the protrusion is bilateral there is nearly always a greater exophthalmos on the side of the greater intracranial pressure or lesion.

2030 Chestnut street.

Diagnosis of Extragenital Chancres.--The main point in the dingmosis of extragenital chancres is to remember that they may occur on any part of the hody; if they are not thought of they will escape diagnosis. The old adluge is here reversed-. it is a case of out of mind, out of sight.-C. F. Marshall, in the l'ruclitioner. 